\title{
Colonización de macroinvertebrados acuáticos en tres tipos de sustratos artificiales, en un río tropical
}

\author{
Francisco Quesada-Alvarado ${ }^{1} \&$ Darha Solano-Ulate ${ }^{2}$ \\ 1. Instituto Regional de Estudios en Sustancias Tóxicas (IRET), Universidad Nacional, Campus Omar Dengo, Heredia, \\ Costa Rica; franqal@gmail.com \\ 2. Universidad de Costa Rica, Escuela de Biología, San Pedro, 11501-2060, San José, Costa Rica; darha.s.u@gmail.com
}

Recibido 23-IV-2019. Corregido 07-VII-2020. Aceptado 31-VIII-2020.

\begin{abstract}
Aquatic macroinvertebrate colonization on three artificial substrates, in a tropical river. Introduction: Different methodologies exist for sampling aquatic macroinvertebrates, however, some of these (e.g., kick-net, Surber) are not well suited for deep, contaminated rivers or rivers with presence of crocodiles, which represent a risk to the person who must take the sample. One alternative is to use artificial substrates. The use of artificial substrates to evaluate the aquatic macroinvertebrate biodiversity in wetlands has been studied previously in Costa Rica, but no research has been conducted to determine which types of substrates are most effective. The present study aimed to evaluate the effectiveness of three artificial substrates (i.e., stones, leaves and wood). Methods: Samples of the three substrates were taken bi-monthly between 2013 and 2017 in four sites of the Parismina River. A Kruskal-Wallis test was applied to determine differences between the macroinvertebrate abundance among substrate type. Beside to determine if there is a difference between substrates per site, a two-way PERMANOVA test was applied and a multiple correspondence analysis was used to correlate the substrate type with the macroinvertebrate community. Results: A total of 1159 organisms were collected, with Diptera and Ephemeroptera being the most abundant. Wood and leaves were the substrates with greatest biodiversity and abundance of organisms; in addition the two sites with greatest vegetation were the most diverse. Conclusions: Most of the organisms were registered on any of the three types of artificial substrates, thus we recommend the joint use of the three types of substrates for biomonitoring or taxa richness studies.
\end{abstract}

Keywords: Insecta; non-wadeable river; colonization; aquatic insects; lowland river.

Quesada-Alvarado, F., \& Solano-Ulate, D. (2020). Colonización de macroinvertebrados acuáticos en tres tipos de sustratos artificiales, en un río tropical. Revista de Biología Tropical, 68(Supl. 2), S68-S78.

La composición del sustrato es una de las características principales que determina el ensamblaje de macroinvertebrados dulceacuícolas en un cuerpo de agua, debido a las diferentes preferencias y adaptaciones de los organismos a este. Así, se espera encontrar cambios en dichos ensamblajes según se modifican las características geomorfológicas del río, desde la cuenca alta hasta la cuenca baja (Czerniawska-Kusza, 2004; Duan, Wang, \& Tian, 2008). En la cuenca alta se observan principalmente sustratos rocosos y heterogéneos, mientras que en las cuencas bajas se suelen observar sustratos arenosos o lodosos inestables para el asentamiento de los organismos bentónicos (Vannote, Minshall, Cummins, Sedell, \& Cushing, 1980; Phillips, 2003). Aunado a esto, los ríos de cuencas bajas se caracterizan generalmente por tener un cauce ancho y profundo que dificulta la recolecta de macroinvertebrados acuáticos presentes en los microhábitats alejados de la ribera (De Pauw, 
Roels, \& Fontoura, 1986; Hernández, Rueda, Tapia, \& Martínez-López 1998; Boothroyd \& Dickie, 2010).

Entre otras dificultades o riesgos para la recolecta de macroinvertebrados acuáticos en las cuencas bajas, se destacan los flujos altos de caudal, presencia de contaminantes, la composición del lecho, la irregularidad del fondo y la presencia de cocodrilos (en regiones tropicales; Hernández et al., 1998). Debido a estas circunstancias, es necesaria la implementación de técnicas que permitan recolectar los macroinvertebrados acuáticos disminuyendo el riesgo para el investigador. Entre las técnicas utilizadas en este tipo de cuerpos de agua, está la aplicación de sustratos artificiales. Esta metodología consiste en colocar en el punto de muestreo trampas compuestas por elementos naturales o artificiales, contenidos en bolsas o cajas con agujeros que permiten la entrada y colonización de los organismos al sustrato elegido (De Pauw et al., 1986; Saliu \& Ovuorie, 2006). Finalizado el período óptimo de 30 a 40 días, las trampas se retiran para el conteo e identificación de los organismos que colonizaron (Hilsenhoff, 1969; Boothroyd \& Dickie, 2010).

Los sustratos artificiales presentan la ventaja de permitir la estandarización de variables como la oferta de sustratos en el cauce, para realizar un análisis comparativo de diferentes secciones de una cuenca, o incluso, entre cuencas independientes, al homogenizar los tipos de microhábitats evaluados. Así, las muestras obtenidas están asociadas en primera instancia a la calidad del agua en el sitio, y no a la disponibilidad del microhábitat (Meier, Penrose, \& Polakf, 1979; Mirto \& Danovaro, 2004; Boothroyd \& Dickie, 2010).

El tipo de sustrato comúnmente utilizado es el rocoso, mediante el uso de ladrillos o bloques de concreto, pues ofrece una rugosidad adecuada para formar refugios para los organismos. Además, permiten la estimación de densidades al proporcionar un área de colonización conocida (Trama, Rizo, \& Springer, 2009). Este sustrato ha mostrado ser efectivo y suele ser recomendado para estudios de biomonitoreo (De Pauw et al., 1986; Czerniawska-Kusza, 2004). Sin embargo, hay una gran variedad de materiales que podrían ser utilizados como sustrato, algunos estudios han evaluado el uso de láminas de acrílico, canicas y plásticos, obteniendo resultados favorables en la colonización de organismos (De Pauw, Lambert, Van Kenhove, \& Bij De Vaate, 1994; CzerniawskaKusza, 2004). No obstante, el uso simultáneo de diferentes materiales, tanto orgánicos como inorgánicos, para la construcción de los sustratos artificiales ha resultado más efectivo pues otorga diversidad de microhábitats para ser colonizados por un mayor número de macroinvertebrados (De Pauw et al., 1986; Pashkevich \& Pavluk, 1996; Phillips, 2003; Molokwu et al., 2014, Leite-Rossi, Nunes, \& Trivinho-Strixino, 2015; Milesi, Dolédec, \& Melo, 2016).

Los estudios con sustratos artificiales en ríos de cuenca baja han sido realizados principalmente en regiones templadas y subtropicales, donde en algunos países la metodología se utiliza para el monitoreo biológico de la calidad del agua (De Pauw et al., 1994; CzerniawskaKusza, 2004; Boothroyd \& Dickie, 2010). Adicionalmente, en la región tropical se han llevado a cabo investigaciones recientes que han probado la importancia de la heterogeneidad de microhábitats en la colonización de macroinvertebrados (Molokwu et al., 2014, Leite-Rossi et al., 2015; Milesi et al., 2016). En Costa Rica, la metodología de sustratos artificiales se ha utilizado en pocos estudios en los que únicamente se ha empleado el sustrato rocoso (Trama et al., 2009), por lo que resulta importante analizar la influencia de otros sustratos, principalmente orgánicos, en la colonización de los macroinvertebrados debido a la aplicabilidad que tiene esta metodología en el monitoreo de ríos de cuenca baja. El objetivo de esta investigación fue evaluar la efectividad de tres tipos de sustratos artificiales en la colonización de macroinvertebrados dulceacuícolas, en la cuenca baja del río Parismina, Costa Rica. 


\section{MATERIALES Y MÉTODOS}

Sitio de estudio: Los muestreos se realizaron de manera bimensual entre los años 2013 y 2017, en el río Parismina, en el sector del Silencio, Guácimo, Limón, Costa Rica. El área de estudio comprende la cuenca baja del río, el cual, se considera como un río estable durante las cuatro épocas del año (seca, lluviosa y transiciones), debido a que el canal tiene forma de U y el fondo está compuesto por arena y limo. Por lo tanto, los muestreos se llevaron a cabo en transporte acuático. El tramo muestreado se encuentra entre los 16 y los $10 \mathrm{msnm}$; y presenta variaciones en cuanto a la composición de la vegetación de ribera, que va desde vegetación nula, mezcla entre árboles y pastizal y bosque en ambas márgenes. El estudio se desarrolló en los sitios del río denominados Silencio $\left(10^{\circ} 14^{\prime} 32.42^{\prime}{ }^{\prime} \mathrm{N} \& 8^{\circ} 29^{\prime} 43.24^{\prime}\right.$ 'W), Desanti $\left(10^{\circ} 15^{\prime} 52.93^{\prime \prime N} \& 83^{\circ} 29^{\prime} 4.63^{\prime \prime} \mathrm{W}\right)$, Jiménez (10¹7'20.30”N \& 83²8'17.91'W) y Quebrada Seca (Q.S) (10¹7'27.72”N \& $\left.83^{\circ} 26^{\prime} 42.30^{\prime \prime} \mathrm{W}\right)$. El sitio Silencio se caracteriza por poseer una menor perturbación en el bosque de ribera, mientras los sitios de Desanti y Jiménez, cuentan con una perturbación media, principalmente por la presencia de potreros. Por último, el sitio Quebrada Seca, con alta perturbación en su ribera, sin presencia de árboles y una matriz de potreros.

Metodología: En los cuatro sitios de muestreo, se emplearon tres tipos de sustratos artificiales compuestos por (1) hojas de sotacaballo (Zygia longifolia; peso total de $20 \mathrm{~g}$ ), (2) madera de sotacaballo (120 g) y (3) canto rodado $(310 \mathrm{~g})$. La madera y las piedras fueron lavadas, para eliminar presencia de contaminantes, mientras que las hojas se adquirieron de los árboles del propio margen del río. Cada material se introdujo en una bolsa de malla con $0.6 \mathrm{~cm}$ de luz de poro (para permitir la entrada de distintas tallas de macroinvertebrados acuáticos), se ataron de forma independiente con una cuerda de nylon de $1.5 \mathrm{~m}$ de largo y se sumergieron (la profundidad del río Parismina varió entre los $0.8 \mathrm{~m}$ y $3 \mathrm{~m}$, según estación del año). En cada sitio se colocó un total de tres réplicas por tipo de sustrato artificial, separadas por $50 \mathrm{~m}$ de distancia entre sí $(150 \mathrm{~m}$ de distancia total) y por un periodo de 30 días, para promover la colonización (Trama et al., 2009), luego del cual se retiraron de forma manual, halando la cuerda lentamente y depositándolos inmediatamente en bandejas para evitar la pérdida de individuos (no se pudo introducir una red o malla para extraer los sustratos debido a la presencia de ramas y raíces sumergidas) .

Posteriormente, se colocaron en bolsas plásticas, para ser transportadas al laboratorio y realizar la búsqueda manual de cada organismo. Los individuos extraídos se depositaron en frascos de vidrio y se preservaron en etanol al $80 \%$ para su identificación a la menor categoría taxonómica posible, usando las claves taxonómicas de Courtney \& Merritt (2008), White \& Rougley (2008), Flowers \& De la Rosa (2010), Ramírez (2010), Springer (2010), mientras que Oligochaeta, Nematoda e Hydrachnidia se identificaron a nivel de orden a través de Roldán (1998).

Análisis estadístico: Para evaluar la diversidad y equitatividad del ensamblaje de macroinvertebrados acuáticos, según cada tipo de sustrato se calculó el índice de ShannonWiener y la dominancia a través del índice de Simpson. Debido a que la abundancia de macroinvertebrados acuáticos por tipo de sustrato no presentó distribución normal, se realizó una prueba Kruskal-Wallis, para determinar si existían diferencias entre la cantidad de individuos registrados por tipo de sustrato y por sitio de muestreo, seguido por la prueba Holm post-hoc para determinar cuáles medias diferían entre ellas. Además, para determinar si existe una diferencia entre los sustratos por sitio, se aplicó una prueba PERMANOVA de dos vías. Por último, se realizó un análisis de correspondencia múltiple (ACM), para determinar la asociación de cada género o familia de macroinvertebrados acuáticos a un tipo de sustrato (madera, hojas o piedra). 


\section{RESULTADOS}

Un total de 1159 individuos fueron recolectados, pertenecientes a 52 géneros de 29 familias, representados en 12 órdenes. Los órdenes Diptera y Ephemeroptera mostraron mayor abundancia de organismos en los tres sustratos artificiales, mientras que Hemiptera y Megaloptera presentaron una menor abundancia (Fig. 1). La familia Chironomidae (Diptera), y los géneros Tricorythodes sp. (Ephemeroptera: Leptohyphidae) y Farrodes sp. (Ephemeroptera: Leptophlebiidae) fueron los taxa más abundantes.

En cuanto a la abundancia por sustratos, la madera de sotacaballo albergó la mayor cantidad de taxa y la mayor abundancia, mientras que, el sustrato de piedras obtuvo el menor registro, tanto en riqueza como en abundancia (Tabla 1). Sin embargo, los tres sustratos tuvieron valores similares respecto al índice de Shanon-Wiener y el de Dominancia. Aun así, se encontró que los sustratos madera y hojas albergan una mayor cantidad de organismos que el sustrato piedra, mientras que, entre los sustratos madera y hojas la cantidad de organismos resulta similar (Kruskal-Wallis: 10.15; $\mathrm{P}<0.05$ ) (Fig. 2 A; Tabla 1).

En el sitio Silencio se obtuvo el mayor valor dentro del índice de Shannon-Weiner, así como la mayor abundancia. Sin embargo, los cuatro sitios evaluados presentaron valores similares de diversidad y sin una clara dominancia entre los géneros encontrados (Tabla 1).

La abundancia de organismos registrados difirió significativamente entre los sitios. Silencio y Jiménez presentaron mayores abundancias respecto a Quebrada Seca y Desanti (Kruskal-Wallis: 10.15; P<0.05; Fig. 2 B). No obstante, no hubo diferencia en la abundancia

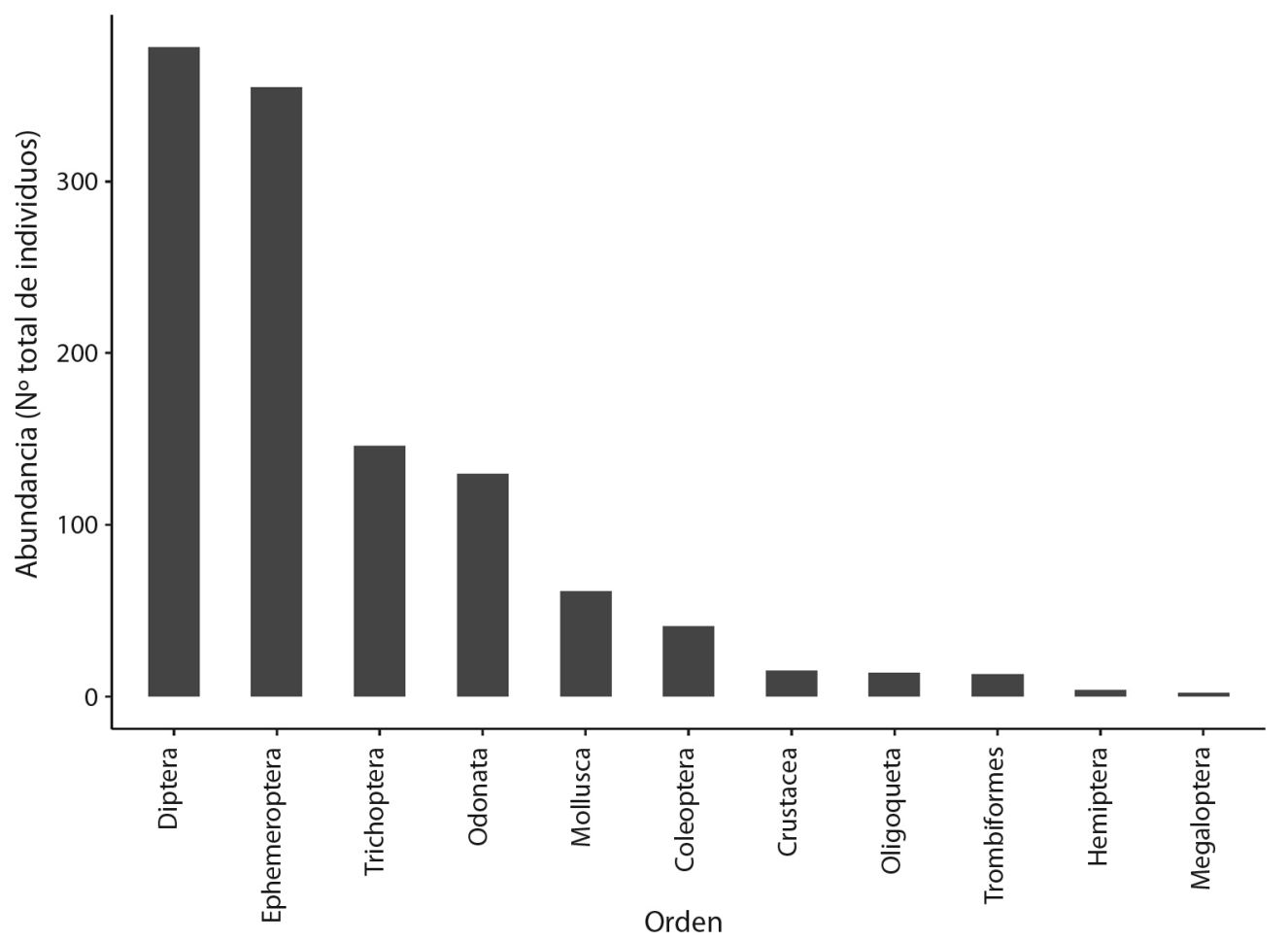

Fig. 1. Abundancia total a nivel de orden de macroinvertebrados acuáticos recolectados en los tres tipos de sustratos artificiales, en los cuatro sitios de muestreo en el Río Parismina, entre los años 2013 y 2017.

Fig. 1. Total abundance at the level of order of aquatic macroinvertebrates collected in three types of artificial substrates, in four sampling sites in the Parismina River, between the years 2013 and 2017. 

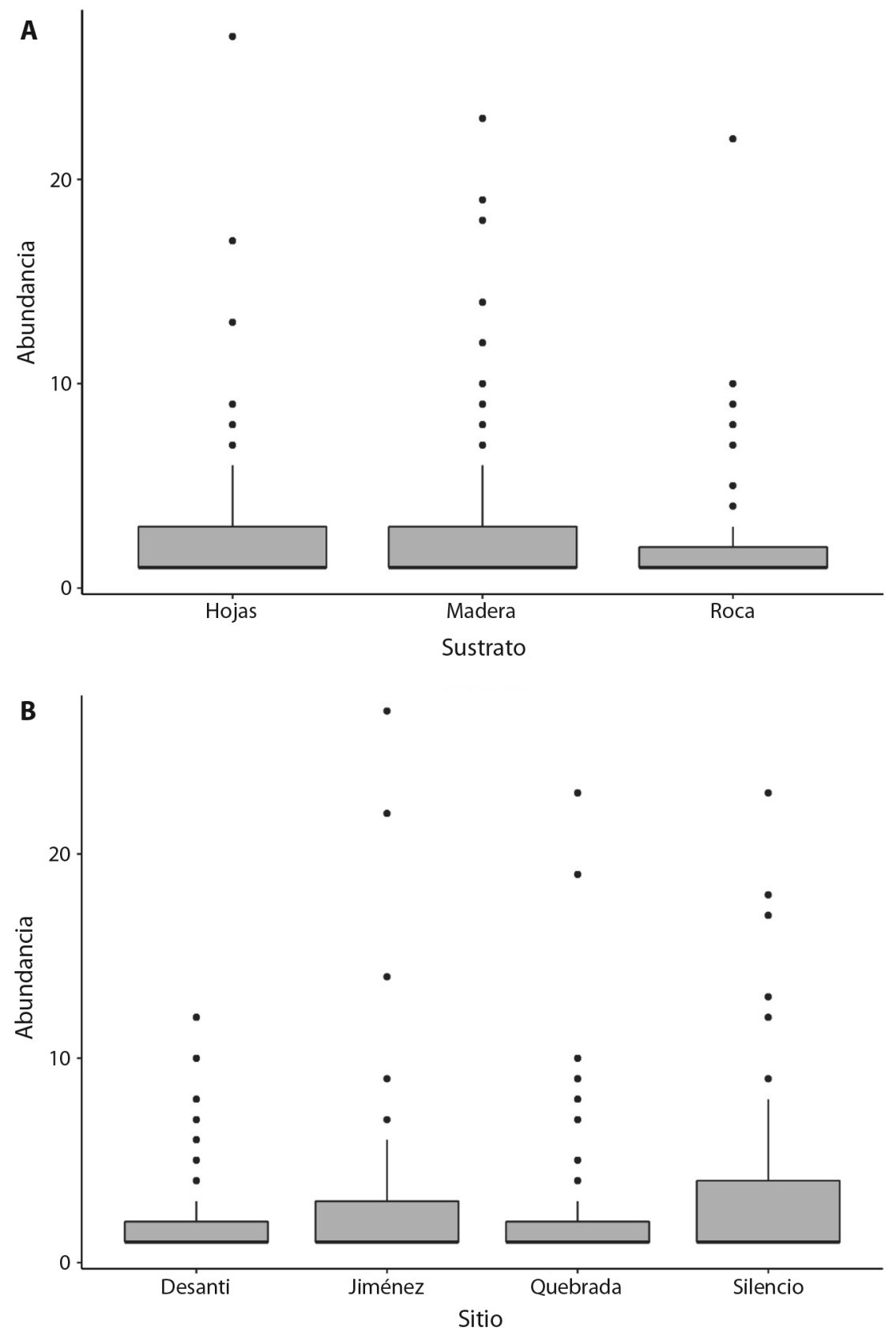

Fig. 2. Abundancia media de macroinvertebrados acuáticos registrados por tipo de sustrato (A), y por sitio (B), en el río Parismina, Costa Rica, entre los años 2013-2017.

Fig. 2. Average abundance of aquatic macroinvertebrate registered by type of substrate (A), and by site (B), Parismina River, between the years 2013 and 2017. 
TABLA 1

Número de taxa, abundancia e índices de diversidad de macroinvertebrados acuáticos, para en tres sustratos artificiales colocados en el río Parismina, entre los años 2013-2017

TABLE 1

Abundance and diversity index of aquatic macroinvertebrates in three artificial substrates in Parismina River, between the years 2013 and 2017

\begin{tabular}{lccccccc} 
& \multicolumn{3}{c}{ Sustratos } & \multicolumn{5}{c}{ Sitios } \\
& Hojas & Madera & Piedra & Desanti & Jiménez & Quebrada Seca & Silencio \\
Taxa & 37 & 42 & 29 & 24 & 27 & 30 & 45 \\
Abundancia & 363 & 494 & 196 & 156 & 184 & 200 & 268 \\
Shannon-Wiener & 3.061 & 3.133 & 2.889 & 2.051 & 2.097 & 2.142 & 2.343 \\
Dominancia & 0.065 & 0.064 & 0.076 & 0.272 & 0.272 & 0.268 & 0.263 \\
\hline
\end{tabular}

entre los sustratos por sitio (Permanova, $\mathrm{F}=$ -11.509, $\mathrm{p}=0.99$ ).

El ACM indicó que la varianza entre los tres tipos de sustratos y los géneros puede ser explicada por los 2 primeros ejes en un $100 \%$ (66.3\% primer eje y $33.7 \%$ segundo eje). El primer eje presentó una relación positiva con los sustratos hojas $(0.51)$ y roca $(0.20)$ y negativa para el sustrato madera (-0.37). Mientras que, el segundo eje presentó una relación positiva para el sustrato roca $(0.62)$ y negativa para los sustratos hojas $(-0.21)$ y madera $(-0.07)$. Algunos géneros tales como, Traverella (Ephemeroptera: Leptophlebiidae), Maccaffertium (Ephemeroptera: Heptageniidae), Oecetis (Trichoptera: Leptoceridae) y Polycentropus (Trichoptera: Polycentropodidae), presentaron una asociación con el sustrato tipo roca. Los géneros Smicridea y Macronema (Trichoptera: Hydropsychidae), Corydalus (Megaloptera: Corydalidae) y Macrelmis (Coleoptera: Elmidae), presentaron mayor asociación con el sustrato tipo hojas. Mientras que, el sustrato tipo madera fue seleccionado por algunos géneros como, Farrodes (Ephemeroptera: Leptophlebiidae), Callibaetis, Americabaetis (Ephemeroptera: Baetidae) y la especie Melanoides tuberculata (Mollusca: Thiaridae) (Fig. 3).

\section{DISCUSIÓN}

En el presente estudio se halló que el uso de sustratos artificiales es una técnica efectiva para la evaluación de la estructura de los ensamblajes de macroinvertebrados acuáticos en sitios con acceso restringido. De los parámetros estudiados, la abundancia fue la característica que mejor diferenció los ensambles, no siendo así la diversidad. La similitud en la diversidad podría deberse a que las condiciones de hábitat y alteraciones son uniformes en el tramo del río estudiado, por lo que no se reflejó la desaparición de organismos sensibles, ni dominancia de organismos tolerantes.

Los sustratos artificiales se colonizaron por una variedad de familias y géneros, sin embargo, la familia más abundante fue Chironomidae. Algunos representantes de la familia Chironomidae se caracterizan por la capacidad de establecerse en una gran variedad de hábitats y en distintas condiciones de la calidad del agua y entorno (Griffith, Kaufmann, Herlihy, \& Hill, 2001; Hedrick et al., 2010; Silva, Ruiz, Bochini, \& Moreira, 2008; Wagner et al., 2008). Similarmente, el género Tricorythodes sp. (Ephemeroptera: Letohyphidae) se registró durante todos los muestreos realizados. Esto podría atribuirse a que, son organismos tolerantes a las perturbaciones del hábitat y pueden sobrevivir en ríos con alta carga de sedimentos (Mesa, 2010). El tercer género más abundante fue Farrodes (Ephemeroptera: Leptophlebiidae). Su flexibilidad alimentaria podría ser una de las razones por las cuales se distribuyó tan ampliamente; este género se alimenta raspando y filtrando (Polegatto \& Froehlich, 2003), por lo que sacan provecho al colonizar los sustratos, 


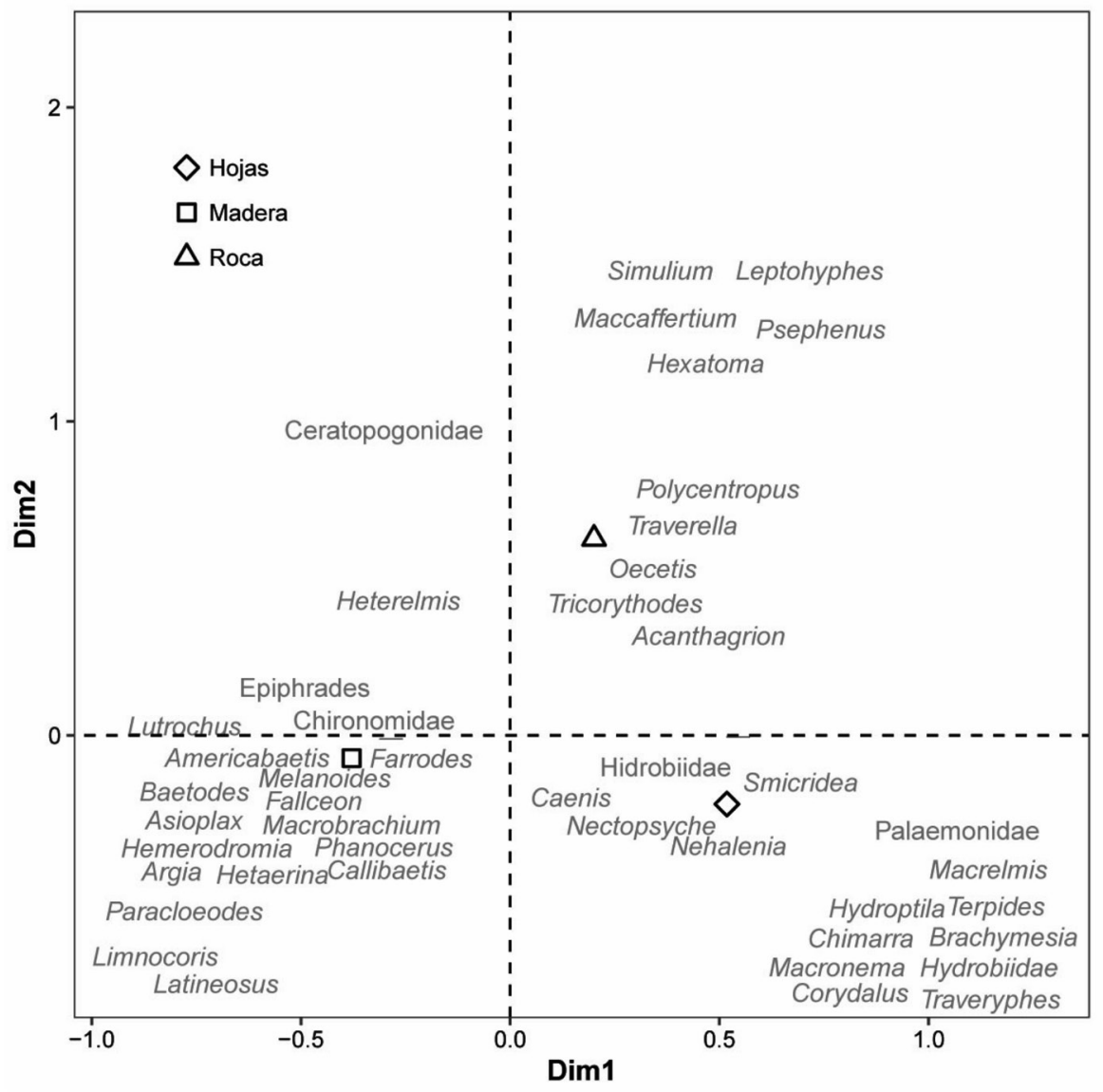

Fig. 3. Análisis de correspondencia múltiple, para los géneros y algunas familias de macroinvertebrados acuáticos según un sustrato artificial, en la cuenca baja del río Parismina. Entre los años 2013 y 2017.

Fig. 3. Multiple correspondence analysis to genera and families of aquatic macroinvertebrates according to an artificial substrate, in Parismina River, Costa Rica. Between the years 2013 and 2017.

ya que pueden raspar el biofilm que se produce o bien en época lluviosa filtrar los sedimentos que acarrea el río, mientras permanecen en un sustrato estable sin ser arrastrados. Por otro lado, la baja abundancia de organismos como Corydalus sp. (Megaloptera: Corydalidae; únicamente dos registros) durante el muestreo, se puede deber a que esta larva prefiere los hábitat rápidos con presencia de canto rodado $\mathrm{y}$ roca y no es común registrarlos en ríos de cuencas bajas con fondo arenoso (Cover \& Resh, 2007). Por lo que se puede tratar de un caso de arrastre por deriva, presencia de una pequeña población en el sector de Silencio donde se obtuvo el registro, o bien, que las larvas no son afines a los sustratos artificiales colocados. Mientras que la baja abundancia en Hemiptera pudo deberse a que estos organismos son nadadores activos y no suele aferrarse a un tipo de sustrato (Zettel, Nieser, \& Polhemus, 1999).

La valores cercanos entre sí del índice de Shannon y de dominancia, dan a entender que las condiciones de hábitat son similatres en el tramo del río estudiado, por lo que no se observó familias y géneros en común entre los sitios de muestreo y $\sin$ dominancia de un organismo sobre otros. En ríos alterados por contaminación o modificación del cauce y ribera, suelen dominar organismos tolerantes a este tipo de modificaciones tales como 
el género Chironomus y la familia Hydrobiidae (Al-shami et al., 2011; Benetti, Perez-Bilbao, \& Garrido, 2012).

Los sustratos artificiales compuestos de madera y hojas, a diferencia de las rocas fueron capaces de albergar una mayor abundancia de macroinvertebrados acuáticos en la cuenca baja del río Parismina. La preferencia de los organismos por los sustratos tipo madera y hojas, puede deberse a que su colonización por microorganismos como hongos, bacteria y algas (Cortes, Abelho, \& Rebelo, 1996; Matt \& Wallace, 1997). Estos conforman una capa que puede ser utilizada como fuente de alimento por algunos macroinvertebrados acuáticos raspadores, como por ejemplo, Farrodes sp., Callibaetis sp., Americabaetis sp. (Ephemeroptera), Heterelmis sp. (Coleoptera) e Hydrobiidae (Mollusca) (Ramírez \& Gutiérrez-Fonseca, 2014), lo cual fomenta a su vez el establecimiento a posteriori de depredadores en el sustrato. La madera, además de la capa de biofilm, genera espacios que brindan refugio a los organismos contra depredadores de mayor tamaño y también por su superficie áspera provee soporte y evita el arrastre (Molokwu et al., 2014; Phillips \& Phillips, 2011). Las hojas también fueron ampliamente colonizadas por distintos macroinvertebrados acuáticos que aprovechan los espacios que se originan y a la vez, son aprovechadas por organismos fragmentadores como Smicridea y Macronema (Trichoptera: Hydropsychidae) (Ramírez \& Gutiérrez-Fonseca, 2014), como se observó en el análisis ACM.

El estudio realizado por Leite et al. (2015), empleó sustratos artificiales compuestos por hojas, madera, esponja Luffa, arcilla expandida y brácteas de pino y observaron que los sustratos compuestos de hojas y madera, presentaron una mayor riqueza y abundandancia en la colonización, ya que son sustratos típicos de los fondos de los ríos, que los macroinvertebrados suelen aprovechar. Por esta razón, es posible que, los macroinvertebrados en el río Parismina hayan presentado en ambos sustratos la mayor abundancia (por encima de las piedras). Sin embargo, a pesar de que el sustrato piedras presentó una menor abundancia y riqueza, en comparación con los otros dos, éste también genera espacios intersticiales, por lo tanto, es un hábitat disponible para que algunos macroinvertebrados acuáticos puedan colonizar (Duan et al., 2008). Se destaca que, el género Traverella (Leptophlebiidae), únicamente se registró en este tipo de sustrato. Este género es abundante en ríos con carga de sedimentos y con presencia de sustrato tipo canto rodado, y al ser un organismo filtrador, aprovecha los espacios intersticiales para protegerse y al mismo tiempo alimentarse (Flowers \& de la Rosa, 2010; Quesada-Alvarado, 2014).

En estudios realizados por Hernández et al. (1998), Czerniawska-Kusza (2004) y Trama et al. (2009) utilizaron únicamente sustrato artificial tipo bloque de concreto y comparados con este estudio se obtuvo valores similares en cuanto a la riqueza de géneros utilizando los tres sustratos. Por otro lado, Kayode y Reubena (2006) utilizaron sustratos artificiales de grava y madera, siendo la grava la que presentó mayor riqueza. Por lo que, la preferencia hacia un sustrato puede depender de la composición de la comunidad o quizás el tipo de cuerpo de agua. Si bien en el presente estudio, no hallamos una fuerte asociación al sustrato piedra, recomendamos el uso del mismo con el propósito de registrar la mayor cantidad de organismos posibles. Sin embargo, cabe destacar que la desventaja de utilizar este tipo de material, es que debido a su peso precipita al lecho o al margen del río y se colmatan con los sedimentos finos que son transportados. Así, sugerimos colocarlos asegurando que éstos no toquen en el lecho, para que mantengan su funcionamiento.

Durante el estudio, se esperaba que las hojas fueran colonizadas principalmente por fragmentadores, la madera por raspadores y las piedras por filtradores. Sin embargo, se registró una mezcla de estos organismos en cada uno de los tres tipos de sustratos (Análisis de Correspondencia Multiple), debido a que cuentan por igual con espacios para refugio, capa de biofilm y soporte para evitar ser arrastrados. Siendo pocos organismos (los géneros Traverella, 
Polycentropus, Corydalus y Limnocoris) los que se registraron en un único tipo de sustrato.

Las diferencias entre organismos registrados por sitio, pueden deberse a la presencia y conformación del bosque de ribera, ya que, en el punto de muestreo Silencio se observa una mayor cantidad de árboles y arbustos en comparación con los otros sitios. Se ha documentado que esta condición suele favorecer una mayor riqueza y abundancia de organismos, debido a que les confiere hábitat para el estadio adulto de los macroinvertebrados acuáticos y sombra, para las larvas y ninfas que se encuentran en el cuerpo de agua (García \& Jiménez, 2003; Arcos, 2006; Harvey, Casanoves, \& León, 2006). Siendo esta una de las posibles razones, que evidencia la diferencia en cuanto a su riqueza y abundancia, entre los sitios Silencio y Quebrada Seca.

En resumen, nuestro estudio demostró que los tres sustratos artificiales en conjunto son efectivos, ya que logran albergar una alta riqueza de macroinvertebrados acuáticos y permiten registrar organismos en sitios que presentan condiciones adversas, o en aquellos donde no se puede caminar con facilidad por ambas márgenes. Por último, se recomienda siempre utilizar hojas y madera típicas de ribera y sin presencia de taninos, que eviten la colonización.

Declaración de ética: los autores declaran que están de acuerdo con esta publicación; que no existe conflicto de interés de ningún tipo; y que ha cumplido con todos los requisitos y procedimientos éticos y legales pertinentes. Todas las fuentes de financiamiento se detallan plena y claramente en la sección de agradecimientos. El respectivo documento legal firmado se encuentra en los archivos de la revista.

\section{AGRADECIMIENTOS}

Un especial agradecimiento a Anny Chaves-Quirós y al Instituto Costarricense de Electricidad, por permitirnos ser parte del proyecto Offset-Parismina. A Iriabel Grant, Kimberly Rojas y Franklin Zamora por formar parte del proyecto. De igual forma a Marvin Matamoros por su constante ayuda en la lancha y búsqueda de los sustratos artificiales. Daniela Vidaurre y Pablo Gutiérrez por las revisiones y recomendaciones sobre el documento. A Jennifer Crowe, Paul Hanson y a Sky Nijman por la revisión del resumen en inglés.

\section{RESUMEN}

Introducción: Existen diferentes metodologías para la recolecta de macroinvertebrados acuáticos (p.e., la red D y Red Surber), sin embargo, algunas de estas no son efectivas en todos los cuerpos de agua o representa un riesgo para la persona que adquiere la muestra. Los sustratos artificiales pueden emplearse en ríos profundos, contaminados o con presencia de cocodrilos. En Costa Rica hay estudios donde se utilizó los sustratos artificiales, para determinar la riqueza y abundancia en humedales tipo lago y lagunas, no obstante se desconoce la efectividad de un tipo de sustrato sobre otro y en sistemas lóticos. El objetivo del estudio fue demostrar la efectividad de tres tipos de sustratos artificiales. Métodos: las muestras se obtuvieron de manera bimensual entre los años 2013 y 2017, en el Río Parismina, Costa Rica, en cuatro sitios a lo largo del río y se evaluó los sustratos piedra, madera y hojas. Se aplicó una prueba de Kruskal-Wallis para determinar las diferencias entre la abundancia y el tipo de sustrato, como también para determinar la diferencia entre la abundancia y el sitio de muestreo. Además, para determinar si existe una diferencia entre los sustratos por sitio, se aplicó una prueba Permanova de dos vías. Un análisis de correspondencia múltiple para determinar el grado de asociación de la comunidad con respecto al tipo de sustrato. Resultados: Un total de 1159 organismos fueron recolectados, siendo Diptera y Ephemeroptera los órdenes más abundantes. La madera y hojas fueron los sustratos con mayor biodiversidad, como también los sitios con mayor vegetación. Conclusiones: El uso en conjunto de los tres tipos de sustratos artificiales, es efectivo para obtener una mayor riqueza de organismos, ya que se observó afinidad por un sustrato según el género de macroinvertebrado acuático.

Palabras clave: Insecta; colonización; insectos acuáticos; cuenca baja.

\section{REFERENCIAS}

Al-shami, S. A., Salmah, C., Hassan, A., Abdul, S., Azizah, S., \& Nor, M. (2011). Ecotoxicology and environmental safety influence of agricultural, industrial, and anthropogenic stresses on the distribution and diversity of macroinvertebrates in Juru River Basin. Ecotoxicology and Environmental Safety, 74(5), 1195-1202. 
Arcos, I. (2006). Efecto del ancho del bosque ribereño en la calidad del agua en la microcuenca del río Sesesmiles, Copán, Honduras: uso de comunidades de macroinvertebrados bentónicos como organismos indicadores. Recursos Naturales y Ambiente, 48(1), 29-34.

Boothroyd, I. K. G., \& Dickie, B. N. (2010). Macroinvertebrate colonisation of perspex artificial substrates for use in biomonitoring studies. New Zeland Journal of Marine and Freshwater Research, 23, 467-478.

Castillo, L. E., Martínez, E., Ruepert, C., Savage, C., Gilek, M., Pinnock, M., \& Solis, E. (2006). Water quality and macroinvertebrate community response following pesticide applications in a banana plantation, Limon, Costa Rica. Science of the Total Environment, 367, 418-432.

Benetti, C., Perez-Bilbao, A., \& Garrido, J. (2012). Macroinvertebrates as indicators of water quality in running waters: 10 years of research in rivers with different degrees of anthropogenic impacts. In K. Voudouris (Ed.), Ecological Water Quality - Water Treatment and Reuse (pp. 23-44). Rijeka, Croatia: InTech

Cortes, R. M. V, Abelho, M., \& Rebelo, S. B. (1996). The macroinvertebrate colonization of leaf Bags: Is there a patterns? Limnetica, 13(2), 71-75.

Courtney, G., \& Merrit, R. (2008). Aquatic Diptera, larvae of aquatic diptera. An introduction to the aquatic insects of North America. Michigan, United States: Kendall/Hunt Publishing Company.

Cover M. R., \& Resh V. H. (2007) Global diversity of dobsonflies, fishflies, and alderflies (Megaloptera; Insecta) and spongillaflies, nevrorthids, and osmylids (Neuroptera; Insecta) in freshwater. Hydrobiology, 198(1), 409-417

Czerniawska-Kusza, I. (2004). Use of artificial substrates for sampling benthic macroinvertebrates in the assessment of water quality of large lowland rivers. Polish Journal of Environmental Studies, 13, 579-584.

De Pauw, N., Roels, D., \& Fontoura, P. (1986). Use of artificial substrates for standardized sampling of macroinvertebrates in the assessment of water quality by the Belgian Biotic Index. Hydrobiologia, 133, 237-258.

De Pauw, N., Lambert, V., Van Kenhove, A., \& Bij De Vaate, A. (1994). Performance of two artificial substrate samplers for macroinvertebrates in biological monitoring of large and deep rivers and canals in Belgium and Netherlands. Environmental Monitoring and Assessment, 30, 25-47.

Duan, X., Wang, Z., \& Tian, S. (2008). Effect of streambed substrate on macroinvertebrate biodiversity.
Frontiers of Environmental Science \& Engineering in China, 2, 122-128.

Flowers, W., \& De la Rosa, C. (2010). Ephemeroptera. Macroinvertebrados de agua dulce de Costa Rica I. Revista de Biología Tropical, 58(Supl. 4), 63-93.

García, L. A., \& Jiménez, F. (2003). Efectos del bosque ribereño y de las actividades antrópicas en las características físico-químicas y en poblaciones de macroinvertebrados acuáticos en la subcuenca del río Tascalapa, Honduras. Resumen. Recursos Naturales y Ambiente, 48(48), 35-46.

Griffith, M. B., Kaufmann, P. R., Herlihy, T., \& Hill, B. H. (2001). Analysis of macroinvertebrate assemblages in relation to environmental gradients in Rocky Mountain streams. Ecological Applications, 11(2), 489-505.

Hammer, Ø., Harper, D., \& Ryan, P.D. (2018). PAST: Paleontological statistics software package for education and data analysis. Palaeontologia Electronica 4(1): 9 .

Harvey, C., Casanoves, F., \& León, J. A. (2006). Efecto del ancho del bosque ribereño en la calidad del agua en la microcuenca del río Sesesmiles, Copán, Honduras. Recursos Naturales y Ambiente 48, 29-34.

Hedrick, L. B., Welsh, S. A., Anderson, J. T., Lin, L. S., Chen, Y., \& Wei, X. (2010). Response of benthic macroinvertebrate communities to highway construction in an Appalachian watershed. Hydrobiologia, 641(1), 115-131.

Hernández, R., Rueda, J., Tapia, G., \& Martínez-López, F. (1998). Efectividad de los substratos artificiales para el muestreo de macroinvertebrados en ríos. Ecología, 12, 151-166.

Hilsenhoff, W. (1969). An artificial substrate device for sampling benthic stream invertebrates. Limnology and Oceanography, 14(3), 465-471.

Oksanen, J. (2019). Multivariate Analysis of Ecological Communities in R: vegan tutorial. Retrieved from http://cc.oulu.fi/ jarioksa/opetus/metodi/vegantutor. pdf

Kayode, J., \& Reubena, U. (2006). The artificial substrate preference of invertebrates in Ogbe Creek, Lagos, Nigeria. Life Science Journal, 4(3), 77-81.

Leite-Rossi, L. A., Nunes, S., \& Trivinho-Strixino, G. (2015). Aquatic macroinvertebrate colonization of artificial substrates in low-order streams. Biotemas, 28(3), 69-77.

Matt, R., \& Wallace, J. B. (1997). Leaf litter decomposition and macroinvertebrate communities in headwater streams draining pine and hardwood catchments. Hydrobiologia 353, 107-119. 
Meier, G., Penrose, D., \& Polak, L. (1979). The rate of colonization by macro-invertebrates on artificial substrate samplers. Freshwater Biology, 9(4), 381-392.

Mesa, L. M. (2010). Effect of spates and land use on macroinvertebrate community in Neotropical Andean streams. Hydrobiologia, 641(1), 85-95.

Milesi, S. V., Dolédec, S., \& Melo, A. S. (2016). Substrate heterogeneity influences the trait composition of stream insect communities: an experimental in situ study. Freshwater Science, 35(4), 1321-1329.

Mirto, S., \& Danovaro, R. (2004). Meiofaunal colonisation on artificial substrates : a tool for biomonitoring the environmental quality on coastal marine systems. Marine Pollution Bulletin, 48, 919-926.

Molokwu, N. D., Vaz P. G., Bradshawa, T., Blake, A., Henessey, C., \& Merten, E. (2014). Effects of substrate on the benthic macroinvertebrate community: An experimental approach. Ecological Engineering, $73,109-114$

Pashkevich, A., \& Pavluk T. (1996). Efficiency of standardized artificial substrate for biological monitoring of river water quality. Environmental Monitoring and Assessment, 40, 143-156.

Phillips, E. C. (2003). Habitat preference of aquatic macroinvertebrates in an East Texas sandy stream. Journal of Freshwater Ecology, 18(1), 1-11.

Phillips, E. C., \& Phillips, E. C. (2011). Habitat Preference of Aquatic Macroinvertebrates in an East Texas Sandy Stream. Freshwater Ecology, 18, 1-11.

Polegatto, C.M., \& Froehlich, C.G. (2003). Feeding strategies in Atalophlebiinae (Ephemeroptera: Leptophlebiidae), with considerations on scraping and filtering. In E. Gaino (Ed.), Research Update on Ephemeroptera \& Plecoptera (pp. 55-61). Italia, Perugia: University of Perugia.

Quesada-Alvarado, F. (2014). Selección y preferencia de hábitat del estado larval de las ninfas de Perlidae (Plecoptera), Corydalidae (Megaloptera) y Leptophlebiidae (Ephemeroptera) como insumo para la determinación de un caudal ambiental en tres represas hidroeléctricas (Tesis de pregrado). Universidad Nacional, Costa Rica.

R Core Team. (2017). R: A language and environment for statistical computing. Version 3.4.2. Vienna, Austria: R Foundation for Statistical Computing.
Ramírez, A. (2010). Odonata. Macroinvertebrados de agua dulce de Costa Rica I. Revista de Biología Tropical, 58(Supl. 4), 63-93.

Ramírez, A., \& Gutiérrez-Fonseca, P. E. (2014). Functional feeding groups of aquatic insect families in Latin America : A critical analysis and review of existing literature. Revista Biología Tropical, 62,155-167.

Roldán, G. (1998). Guía para el estudio de los macroinvertebrados acuáticos del Departamento de Antioquia. Bogotá, Colombia: Pama Editores Ltda.

Saliu, J. K., \& Ovuorie, U. R. (2006). The artificial substrate preference of invertebrates in Ogbe Creek, Lagos, Nigeria. Life Science Journal, 4, 77-81.

Silva, F. L., Ruiz, S. S., Bochini, G. L., \& Moreira, D. C. (2008). Functional feeding habits of Chironomidae larvae (Insecta, Diptera) in a lotic system from Midwestern region of Sao Paulo State, Brazil. PanAmerican Journal of Aquatic Sciences, 3(2), 135-141.

Springer, M. (2010). Trichoptera. Macroinvertebrados de agua dulce de Costa Rica I. Revista de Biología Tropical, 58(Supl. 4), 63-93.

Trama, F. A., Rizo Patrón V, F. L., \& Springer, M. (2009). Macroinvertebrados bentónicos del humedal de Palo Verde, Costa Rica. Revista de Biología Tropical, 57(1), 275-284.

Vannote, R.L., Minshall, G.W., Cummins, K.W., Sedell, K.W. \& Cushing, C.E. (1980). The river continnum concept. Canadian Journal of Fisheries and Aquatic Sciences, 37,130-137.

Wagner, R., Barták, M., Borkent, A., Courtney, G., Goddeeris, B., Haenni, J. P., ... Zwick, P. (2008). Global diversity of dipteran families (Insecta Diptera) in freshwater (excluding Simulidae, Culicidae, Chironomidae, Tipulidae and Tabanidae). Hydrobiologia, 595(1), 489-519.

White, D., \& Roughley, R. (2008). Aquatic coleopteran. An introduction to the aquatic insects of North America. Michigan, United States: Kendall/Hunt Publishing Company.

Zettel, H., Nieser, N., \& Polhemus D. 1999. The Naucoridae (Insecta: Heteroptera) of the Philipinne Islands. Annalen des Naturhistorischen Museums in Wien, 101, 43-105. 